\title{
Fuzzy Modeling for Quality Estimation of Fading Channel
}

\author{
Maheshwari Niranjan \\ Department of Information Technology \\ Technocrats Institute of Technology \\ Bhopal, India
}

\author{
Amit Sinhal \\ Department of Information Technology \\ Technocrats Institute of Technology \\ Bhopal, India
}

\begin{abstract}
The characterization and modeling of the fading channel are essential to wireless communication design. In this paper, we design a fuzzy-based approach for performing the quality estimation of single-path as well as multi-path fading channels. We have used Rayleigh distribution to create the wireless channel. In addition, certain attributes of the channel (bit error rate, energy, and distance between sender and receiver nodes) are fuzzified with the help of Mamdani method to determine the channel quality. We have also carried out case study of five Rayleigh channels having different characteristics. On the basis of its resultant outcome, we have further presented the suitability of the corresponding channels to voice, video and text transmission.
\end{abstract}

\section{General Terms}

Bit Error Rate, Channel Noise, Fuzzy Channel Modeling

\section{Keywords}

Single-path \& Multi-path Fading Channel, Channel Quality Estimation, Additive White Gaussian Noise

\section{INTRODUCTION}

In wireless sensor networking, the data transmission between nodes is performed through single-hop and multi-hop channels. For such wireless communication, two types of fading channels, namely Rayleigh and Rician are widely used that suitably model the real-world phenomena [3-5]. These phenomena include multipath scattering effects, Doppler shifts, signal strength and time dispersion.

Communication through multi-hop path results in the arrival of delayed versions of the signal at the destination node. Moreover, the radio signal further undergoes scattering effect on local scale for each multi-hop path. Such type of scattering is characterized by large number of reflections by sensor nodes present in close proximity to the receiving node. These irresolvable components combine at the receiving end and results in multipath fading. Due to this phenomenon, each multi-path acts as a discrete fading path. Rayleigh distribution is used for reflected path (i.e. when the destination node can be reached via single/multiple paths) and Rician distribution applied when the receiving node comes directly in the line-ofsight path.

In this paper, we design a fuzzy-based approach for performing the quality estimation of single-path as well as multi-path fading channels. We have used Rayleigh distribution to create the wireless channel. In addition, certain attributes of the channel (bit error rate, energy, and distance between sender and receiver nodes) are fuzzified with the help of Mamdani method to determine the channel quality. We have also carried out case study of five Rayleigh channels having different characteristics. On the basis of its resultant outcome, we have further presented the suitability of the corresponding channels to voice, video and text transmission.

\section{RELATED WORK}

The significance of wireless fading channels plays a vital role in wireless communication. Determination of channel noise in the wireless channel helps in allocating resources and selecting a suitable transmission policy in order to match the instantaneous conditions of the fading channel. There are several important research carried out in the related field. Hence, in this section we have performed a brief review on the previous work and then present our research proposal.

In [1] the authors have presented fading channel model with the help of variable-length Markov Chain. The KullbackLeibler distance is employed as the optimization criteria for approximating certain channel characteristics, for instance average fade duration, signal outage probability, etc. The proposed scheme was found to provide optimal values of channel components by using the Lagrangian optimization. In another contribution [2], the author has tried to highlight the importance of Channel State Information (CSI) for determining the channel quality prior to data transmission over the fading channel. Such quality prediction is often found to achieve significant performance gain in wireless communication.

Another subsequent contribution in [3] used the properties of the Markov chain for characterizing parameters of the fading channel, for e.g. signal fade duration and level crossing rate. The Markov sates are based on a threshold value which is defined as a function of channel parameters - signal-to-noise (SNR) ratio and Doppler frequency. Moreover, the proposed model is also used to derive an analytical expression for the average bit error probability over a Rayleigh fading channel. In [4] the authors have proposed a multipath fading model to analyze the bit error rate of the channel link. The model is further used to obtain channel quality estimation by integrating shadow fading, channel noise and multi-path fading. Further, in [5] it has been shown that at very high signal-to-noise ratio, the sum-rate capacity of a non-coherent multiple-access fading channel is limited by the capacity of the better of the two users.

Recent research carried out in [6] explored a novel way of simulating the behavior of the first order and second order statistic properties of wireless fading channel. The reliability of the proposed method was established by comparing the channel statistical properties with the theoretical model. In yet another contribution [7], the estimation and equalization of multipath fading channel id performed with fuzzy approach. 


\section{PROPOSED FUZZY MODEL FOR WIRELESS CHANNEL}

We have proposed a fuzzy model for estimating the wireless channel quality in terms of channel noise and its suitability to voice, video and text transmission. Our proposed model is based on Mamdani Fuzzy Method with three fuzzified input parameters - Bit Error Rate (BER), residual sensor energy and distance between sender and receiver nodes (fig. 1). Owing to the smoothness of variation in BER and residual energy values, we have employed Gaussian membership function $f_{\text {gauss }}(x)$ and Generalized Bell $f_{\text {gbell }}(x)$ membership function. These functions assist in capturing the continuity of parametric values. The above mentioned membership functions are defined as:

$$
\begin{aligned}
& f_{\text {gauss }}(x)=\exp \left(\frac{-0.5\left(x-c_{\text {gauss }}\right)^{2}}{\sigma^{2}}\right) \\
& f_{\text {gbell }}(x)=\frac{1}{\left(1+\left|\frac{x-c_{\text {gbell }}}{v}\right|^{2 w}\right)}
\end{aligned}
$$

In equations (1-2) $c_{\text {gauss }}$ and $c_{\text {gbell }}$ represent the mean or the center position of the curve for the respective membership functions. Also, $v$ and $w$ are regarded as the width controllers of the Generalized Bell curve. Furthermore, we have assumed distance to be a discrete parameter and therefore used Trapezoidal membership functions. The equation of the Trapezoidal curve is given by the following equation:

$$
f_{\text {trap }}(x)=\left\{\begin{array}{cc}
\frac{0}{x-a} & x<a \\
b-a & a \leq x \leq b \\
1 & b \leq x \leq c \\
\frac{d-x}{d-c} & c \leq x \leq d \\
0 & d \leq x
\end{array}\right.
$$

In above equation, $a$ and $d$ adjust the base of the trapezoid. Moreover, the symbols $b$ and $c$ are used to locate the top portion of the trapezoidal curve. The initial values for all the membership functions of the corresponding input parameters are provided in Table 1-3 respectively.

On the basis of these fuzzy input parameters and fuzzy rules (Appendix A), we derive the fuzzy channel noise. A channel vulnerable to different degrees of noise exhibits whether or not it is suitable for low-level and/or high level transmission. Low-level includes textual data transmission while high-level involves voice and video communication (highly sensitive information).

Table 1. Fuzzy Variable: Bit Error Rate (Gaussian MF)

\begin{tabular}{|c|c|c|}
\hline $\begin{array}{c}\text { Fuzzy } \\
\text { Function }\end{array}$ & $c_{\text {gauss }}$ & $\sigma$ \\
\hline Low & $6.939 \mathrm{e}-018$ & 0.1699 \\
\hline Medium & 0.5 & 0.1698 \\
\hline High & 1 & 0.1692 \\
\hline
\end{tabular}

Table 2. Fuzzy Variable: Residual Energy (Generalized Bell MF)

\begin{tabular}{|c|c|c|c|}
\hline $\begin{array}{c}\text { Fuzzy } \\
\text { Function }\end{array}$ & $c_{\text {gbell }}$ & $v$ & $w$ \\
\hline Less & $6.939 \mathrm{e}-018$ & 0.21 & 2.52 \\
\hline Average & 0.5 & 0.24 & 2.56 \\
\hline More & 1 & 0.22 & $2 / 33$ \\
\hline
\end{tabular}

Table 3. Fuzzy Variable: Distance (Trapezoidal MF)

\begin{tabular}{|c|c|c|c|c|}
\hline $\begin{array}{c}\text { Fuzzy } \\
\text { Function }\end{array}$ & $a$ & $b$ & $c$ & $d$ \\
\hline Near & -0.36 & -0.04 & 0.04 & 0.36 \\
\hline Moderate & 0.1374 & 0.4574 & 0.5374 & 0.8574 \\
\hline Far & 0.64 & 0.96 & 1.04 & 1.36 \\
\hline
\end{tabular}

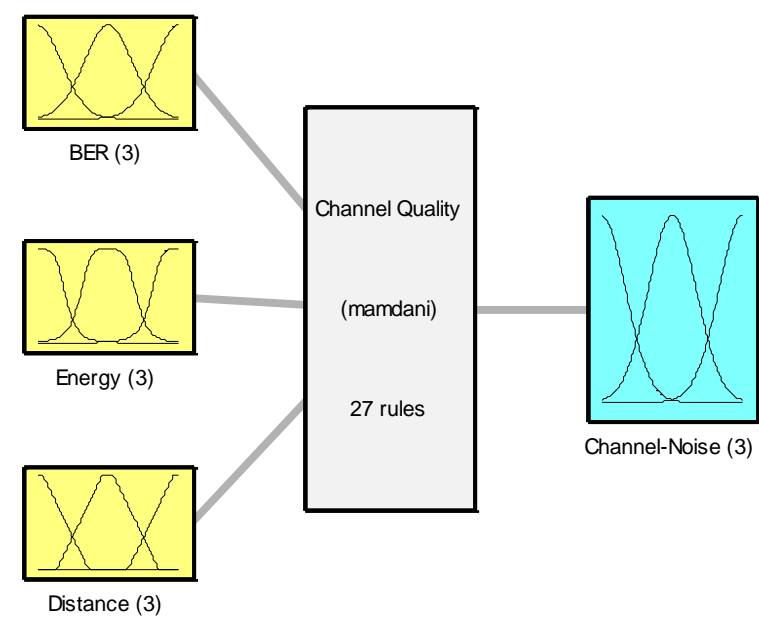

Fig 1: Fuzzy Model for Channel Quality Estimation

\section{QUALITY ESTIMATION OF FADING CHANNEL}

The channel quality estimation is a research of great significance as it helps to assess the pertinence of the channel prior to actual transmission. For instance, if the channel quality is estimated in advance to be poor, one would not prefer to use the specific channel for transmitting highly sensitive data. This saves huge amount of transmission energy and avoids the corruption of the highly sensitive information due to channel noise.

In this project, we have performed the evaluation of different channel parameters that affects the channel quality. For our simulation (in MATLAB) we have considered both the singlepath and multi-path fading channel. We have also considered differences in theoretical and empirical Bit Error Rate of the fading channels. Initially, we generate the random signal with the help of randint function. This signal is further modulated with DPSK modulator. The modulated DPSK signal stream is passed through the Rayleigh channel with Gaussian noise. At the receiving end, the signal is demodulated with DPSK demodulator and consecutively computed the bit error rate of the channel. Also, we have used channel visualization tool to highlighting the bandwidth impulse response, frequency 
response, multipath fading components, and Doppler spectrum. In addition, we have performed a detailed case study of five different Rayleigh Channels with different channel bit rate, sampling period, maximum Doppler shifts and path delay vector. Our proposed fuzzy model is further used to assess the suitability of the channels for different types of communication.

\section{SIMULATION STUDY}

In this section, we highlight the experimental outcomes of our simulation performed in MATLAB. The performance evaluation is carried out separately for the single-path and multi-path fading channel. Additionally, case studies are made regarding the channel suitability for specific services.

\subsection{Single-Path Fading Channel}

In single-path fading channel, the chances of signal scattering is less. This is illustrated by fig. 2 , in which the density of the signal variation is found to be small. Further, in fig. 3 our theoretical fuzzy model the faded channel appears to give fairly good estimation to the empirical value. Fig. 4-5 shows the outcome from the channel visualization simulation, in terms of impulse response and Doppler spectrum.

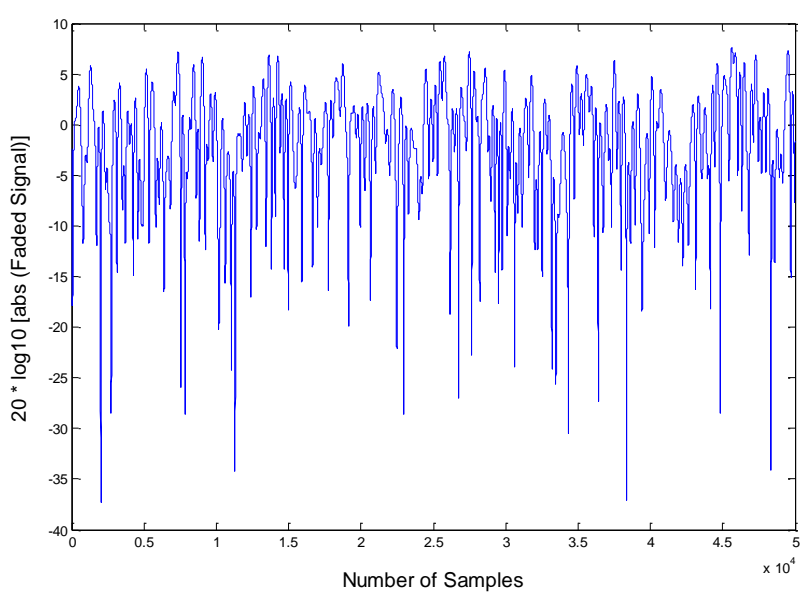

Fig 2: Fading Signal for Single-Path Channel Model

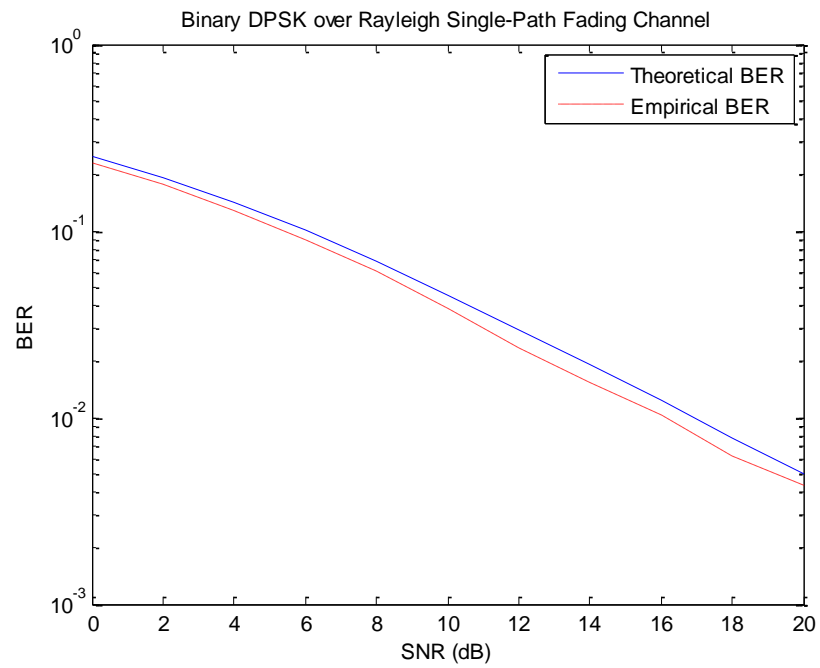

Fig 3: Theoretical BER vs. Empirical BER over SNR

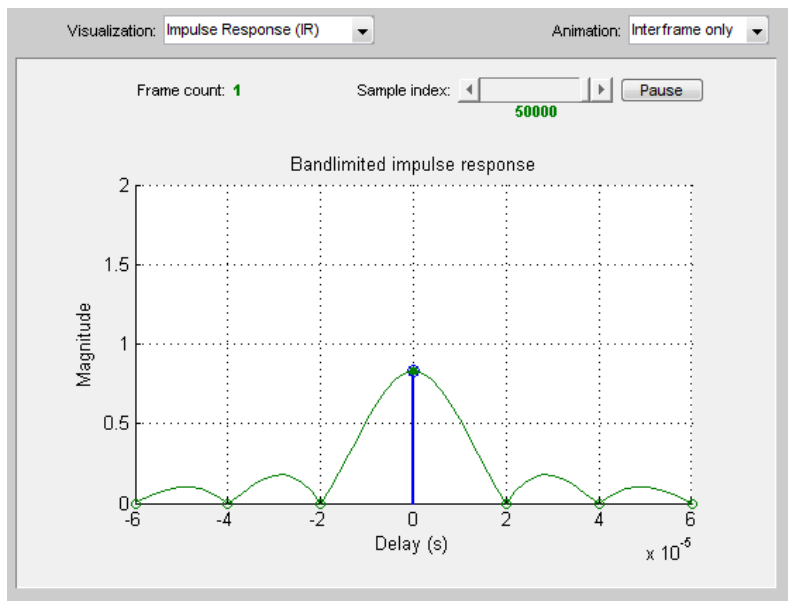

Fig 4: Bandwidth Impulse Response Simulation

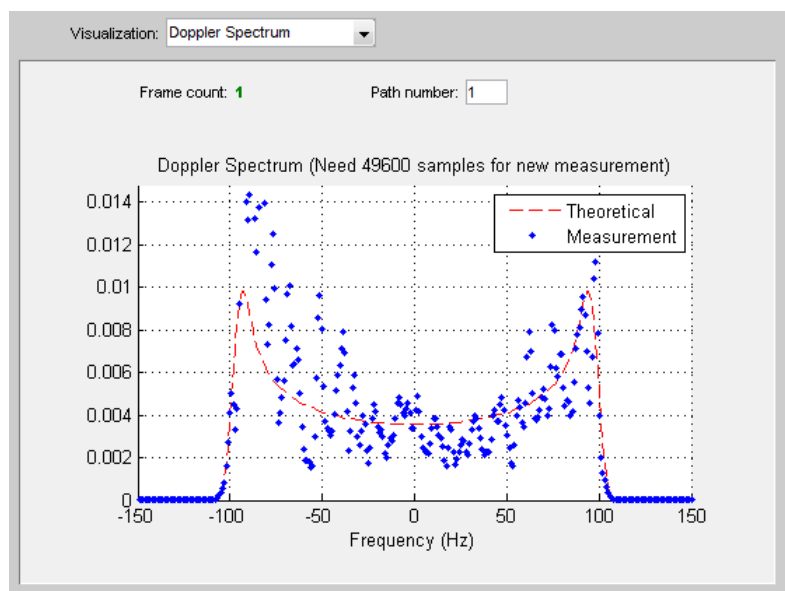

Fig 5: Visualization of Doppler Spectrum over Frequency

The statistical outcome of the proposed fuzzy model of singlepath fading channel is given in fig. 6 . The fuzzified degree of channel noise is derived on the basis of the fuzzy rules and statistics (Appendix A).

\section{Experimental Outcome}

Channel Type: Single-Path Fading Channel

Number of Error Bits: 217

Bit Error Rate (BER): 0.0043

Fuzzified Degree of Channel Noise: 0.4139

Fig 6: Summarized Output of Single-Path Fading Channel 


\subsection{Multi-Path Fading Channel}

The signal fading is found to be comparatively more in the multi-path than the single-path channel respectively. This is shown by increased density in the signal level in fig. 7 .

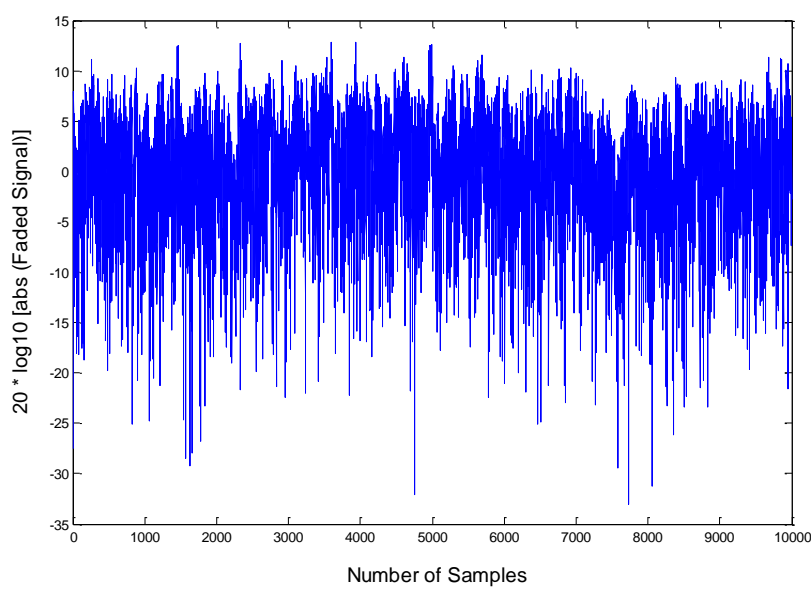

Fig 7: Fading Signal for Multi-Path Channel Model

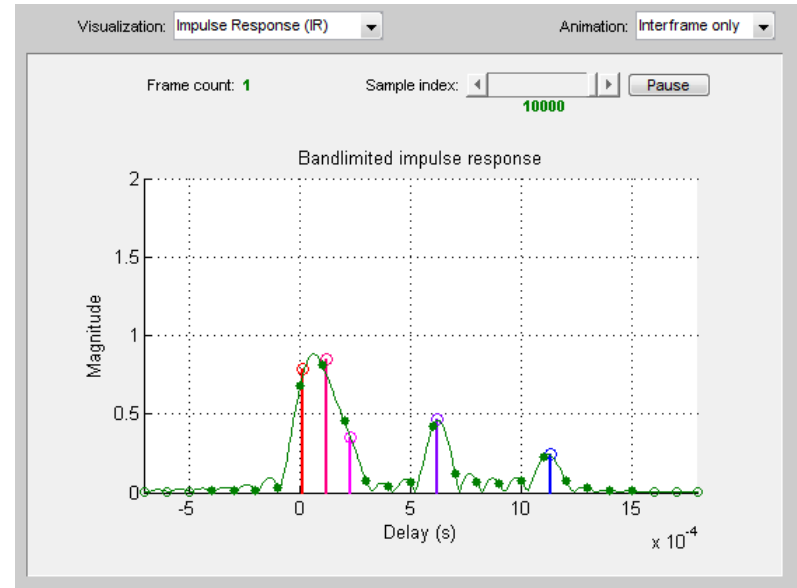

Fig 8: Bandwidth Impulse Response for Multi-Path Channel Visualization

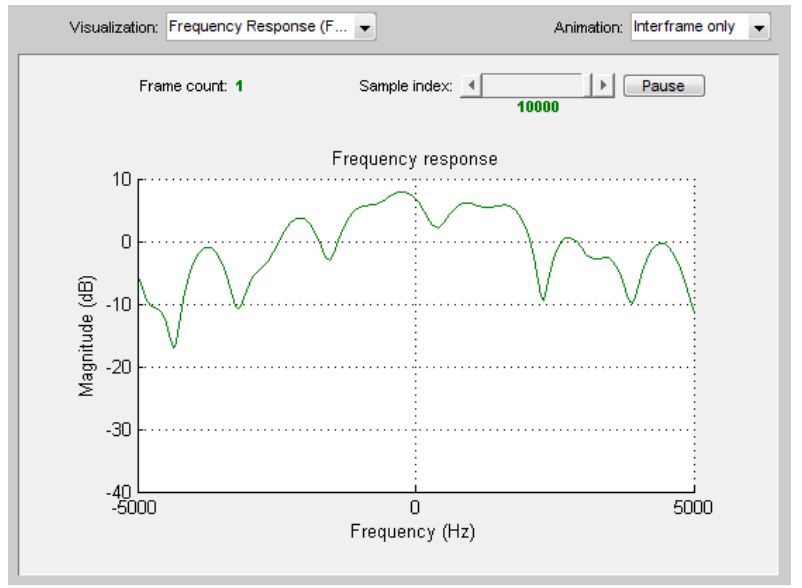

Fig 9: Fuzzy Model for Channel Quality Estimation

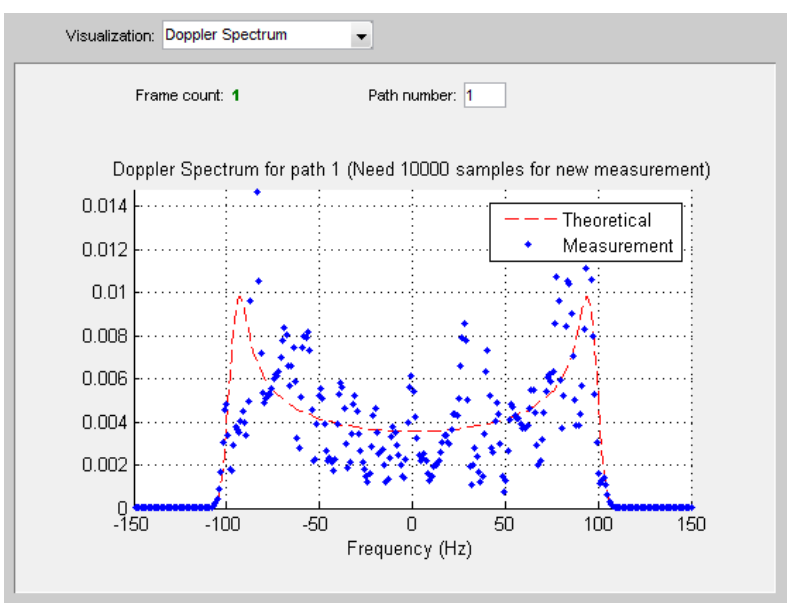

Fig 10: Doppler Spectrum for Multi-Path Fading Channel

In figures $8-10$, simulation results from channel visualization simulation is provided. The plot in fig. 8 shows the magnitudes of multipath response and bandlimited channel response. The stems represent the multipath response each of which corresponds to a multipath component. The smallest delay component is highlighted in red and the largest delay component is shown in blue. Further, in fig. 9 the magnitude of frequency response of the multipath channel is illustrated in decibels over the signal bandwidth. The frequency response changes as the simulation time advances.

In fig. 10 the theoretical and practically measured values of Doppler spectra are highlighted. For the simulation of multipath channel model, we have employed Jakes spectrum as the theoretical spectrum. This plot is based on the filter response due to maximum Doppler shift used in our multipath channel. The second plot of Doppler spectrum is the measured value of the power spectrum of the multipath fading channel as the model generates path gains.

Finally, the experimental result of the proposed fuzzy model of multi-path fading channel is given in fig. 11. It is clarified that the bit error rate is much more in the case of multi-path fading than the single-path model, and hence the channel noise is higher.

Experimental Outcome

Channel Type: Multi-Path Fading Channel

Number of Error Bits: 7299

Bit Error Rate (BER) : 0.3651

Fuzzified Degree of Channel Noise: 0.4863 


\subsection{Case study}

This section deals with the results obtained from our case study of five multi-path fading Rayleigh channels. Each of the channels possesses different properties. From the statistical result given in fig. 12, it can be observed that for lower value of Bit Error Rate (BER), the channel noise is tolerable enough to support audio, video and text transmission. However, with elevation of BER above the predefined threshold value, results in higher degree of channel noise. As a result of which the channel becomes vulnerable to signal distortion. Therefore, in such cases our proposed model recommends the transmission of less sensitive signals, i.e. text data. The audio and more significantly video communication are highly sensitive and hence require less noisy channel to transmit.

\section{CONCLUSION}

Our proposed strategy helps to detect the channel noise prior to transmission of data or signal over the wireless medium. Having knowledge of the noise level in advance further assists the engineers to determine the suitability of the channel with respect to certain applications. Such service applications often include sensitive data like audio and video signals, while other may involve less sensitive data like text. Therefore, if the channel is found to be noisy beyond the threshold limit, then the user can use the channel for textual data and vice versa.

We have also performed the simulation of the proposed fuzzy channel model for both, single-path and multipath respectively. The results from channel visualization are also highlighted along with statistical outcomes.

\section{REFERENCES}

[1] Kumwilaisak, W., Jay Kuo, C.-C. and Wu, D., "Fading Channel Modeling via Variable-Length Markov Chain Technique", IEEE Transactions on Vehicular Technology, vol. 57, no. 3, 2008, pp. 1338-1358.

[2] Duel-Hallen, A., "Fading Channel Prediction for Mobile Radio Adaptive Transmission Systems" Proceedings of the IEEE, vol. 95, no. 12, 2007, pp. 2299-2313.

[3] Dehnie S., "Markov Chain Approximation of Rayeleigh Fading Channel", IEEE International Conference on Signal Processing and Communications (ICSPC 2007), Dubai, United Arab Emirates, 2007, pp. 1311-1314.

[4] Chao S., Bai C., Ying Z., Xinlin W., Lipei, G. and Bin, L., "Multi-Path Fading Channel-Based Estimating Model of Link Quality for Wireless Sensor Networks", IEEE, 2009, pp.1-5.

[5] Lin, G-R. and Moser, S.M., "The Fading Number of a Multiple-Access Rician Fading Channel”, IEEE Information Theory Workshop, 2009, pp. 268-272.

[6] Jun, G.X. and Feng, Z.G., "A Simulation method for Nakagami Fading Channel", $14^{\text {th }}$ International Symposium on Antenna Technology and Applied Electromagnetics and the American Electromagnetics (AMEREM) Conference, 2010, pp. 1-4.

[7] Chen, B-S., Yang, C-Y. and Liao, W-J., "Robust Fast Time-Varying Multipath Fading Channel Estimation and Equalization for MIMO-OFDM Systems via a Fuzzy Method", IEEE Transactions on Vehicular Technology, vol. 61 , no. 4, 2012, pp. 1599-1609.

\begin{tabular}{|c|c|c|c|c|}
\hline & BER & Channel Noise & Noise Level & Suitability \\
\hline & --- & ------------- & ---------- & ---------- \\
\hline Channe 1 1: & 0.3648 & 0.4862 & Medium & Audio/Text \\
\hline Channel 2: & 0.3772 & 0.4895 & High & Text \\
\hline Channel 3: & 0.2469 & 0.4177 & Low & Audio/Video/Text \\
\hline Channel 4: & 0.3742 & 0.4887 & High & Text \\
\hline Channel 5: & 0.4977 & 0.5000 & High & Text \\
\hline
\end{tabular}

Fig 12: Statistical Output for Case Study of 5 Multi-Path Rayleigh Fading Channel 


\section{APPENDIX A}

In order to design our proposed fuzzy model of fading channel, we have used the FIS editor of MATLAB. The fuzzified model is based on Mamdani method, with three fuzzy inputs - Bit Error Rate (BER), distance and residual energy. Our proposed model processes these inputs with the help of rule base (fig. 16) and produces appropriate degree of channel noise for a given single-path or multi-path fading channel.

In fig. 13-15, the surface viewer highlights three dimensional curves that exhibit relationship between the fuzzy input variables (taken in pair) with the fuzzy output variable. Apparently, as BER and distance (between sender and receiver nodes) increases, the channel noise also elevates. Moreover, decrease in the residual energy of the sensor node increases the probability of noise in the channel.

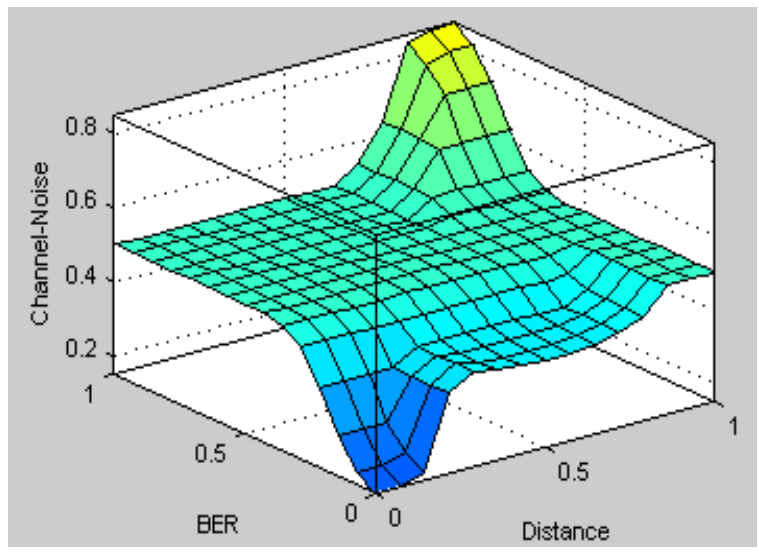

Fig 13: Channel Noise over BER \& Distance

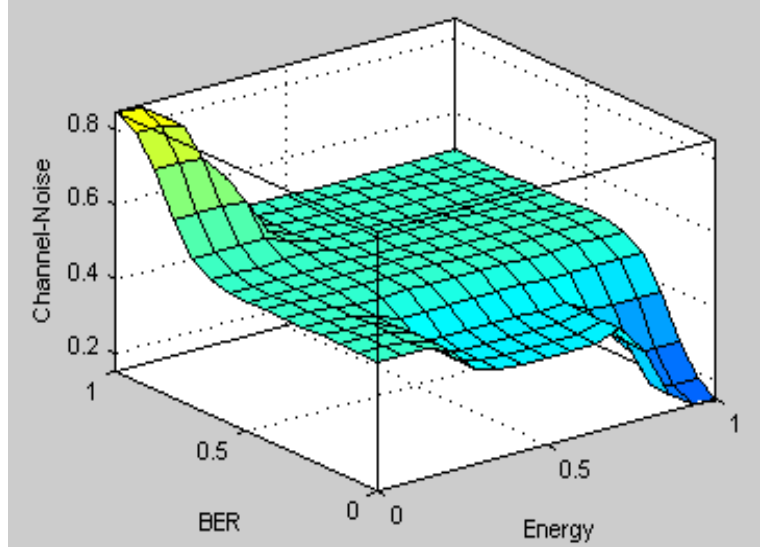

Fig 14: Channel Noise over BER \& Sensor Energy

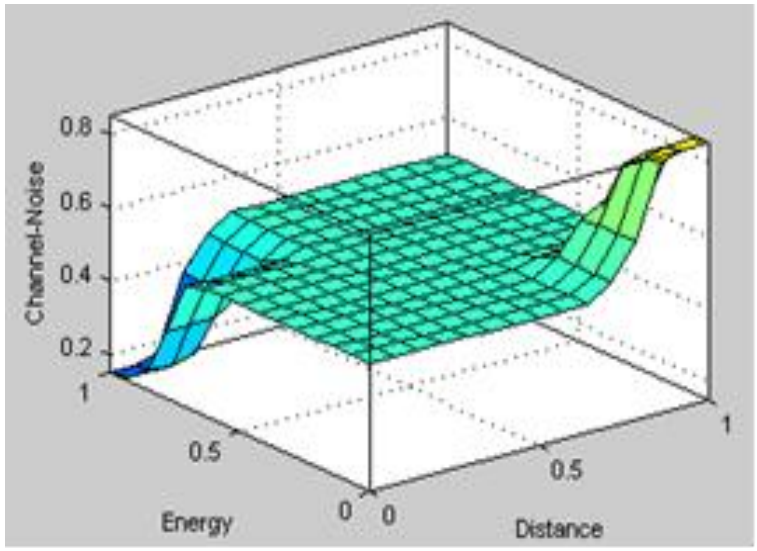

Fig 15: Channel Noise over Sensor Energy \& Distance

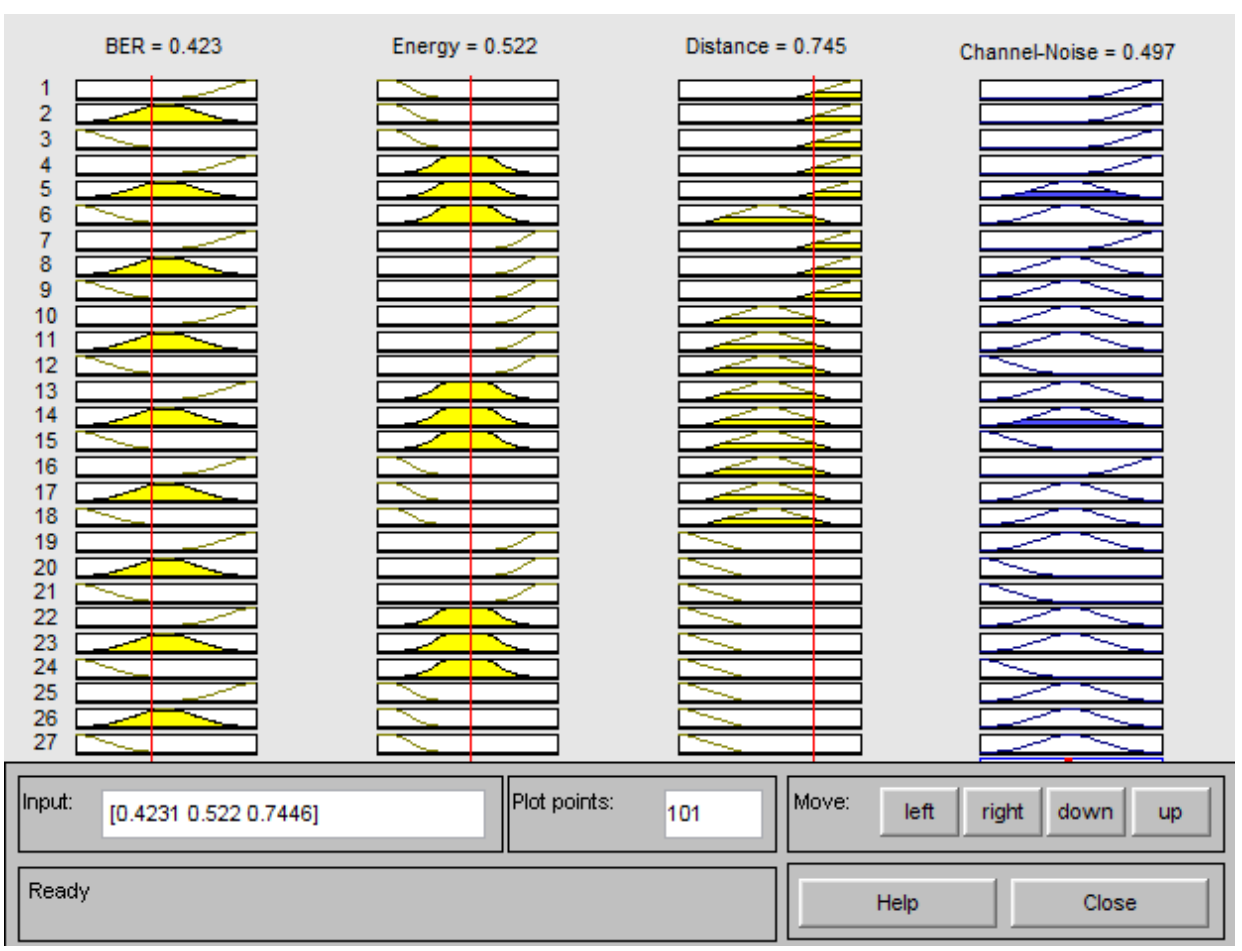

Fig 16: Fuzzy Rule Base for Determination of Appropriate Degree of Channel Noise 